\title{
Nurtured to follow the crowd: A twin study on conformity
}

\author{
LI XueTing, ZHANG JieDong, HUANG Yi, XU Miao \& LIU Jia* \\ State Key Laboratory of Cognitive Neuroscience and Learning, Beijing Normal University, Beijing 100875, China
}

Received September 27, 2012; accepted November 30, 2012

\begin{abstract}
Why are some of us easily affected by the majority's action whereas others frequently make unique choices? In this behavioral genetic study on conformity, we designed a social episode to investigate the genetic and environmental origin of individual differences in conforming behavior during psychosocial development. Specifically, 107 monozygotic (MZ) and 74 dizygotic (DZ) twin pairs (age 7-19) were asked to choose a pen from a group of pens either with the majority color or with the minority color. We found that the resemblance between MZ twins in selecting the pen with the majority color was not significantly higher than that between DZ twins, suggesting that individual differences in conformity are due almost exclusively to the environment. Moreover, biometric model fitting revealed that the shared environmental component played a sizable role in shaping individuals' conforming behavior, and the influence increased with age (from $16 \%$ to $26 \%$ ). Taken together, our study suggests that the common family and cultural environment has important ramifications for conformity.
\end{abstract}

conformity, twin design, nature and nurture, psychosocial development, shared environmental influence

Citation: $\quad$ Li X T, Zhang J D, Huang Y, et al. Nurtured to follow the crowd: A twin study on conformity. Chin Sci Bull, 2013, 58: 1175-1180, doi: 10.1007/ s11434-013-5701-x

Imagine a situation in which a person orders a steak at a restaurant but a waiter kindly informs that the lamb is the best choice. Accordingly, this person may change the order to the lamb, the dish that most customers order, or he or she may stick with the original choice. In this social situation, ordering a dish is about much more than satisfying hunger or taste. Rather, it is a struggle to make a decision in accordance with internal judgment under external pressures to do otherwise (i.e., conformity). Previous studies have demonstrated significant individual differences in conforming behavior [1-3], and the variance is thought to arise from environmental influences, such as parenting style [4] and cultural context [5]. However, little is known about the role of genetic influences, the flip-side of environmental influences, on conformity. In this study, we used the behavioral genetics approach to address two fundamental questions in social influences: (1) Is there any genetic component in conforming behavior; and (2) which environmental component, shared or nonshared experiences, plays a more im-

*Corresponding author (email: liujia@bnu.edu.cn) portant role in shaping conforming behavior during psychosocial development.

Conformity is not just acting as other people act; instead, it is critical in social interaction for achieving social approval of others, building intimate relationships, enhancing self-esteem and for avoiding punishment for violating the norm [6,7]. However, conforming behavior differs significantly across individuals, which is under influences from the social environment surrounding individuals. For example, when asked to choose a pen out of a group of pens of two colors-one color in the majority and the other in the minority, individuals from different cultures show a distinct pattern of choices. Those from collectivistic culture (e.g., Chinese) prefer to choose a pen characterized by the majority (i.e., conformity), whereas those from individualistic culture (e.g., Americans) prefer to choose a pen associated with uniqueness [5].

In contrast to the multitude of studies focusing on the environmental influences on conformity, little is known about the genetic origin. Here we used a behavioral genetics approach to address this question. The most common design in 
behavioral genetics is to compare the resemblance of monozygotic (MZ) twins versus that of dizygotic (DZ) twins to estimate the heritability of the phenotype tested [8]. Because MZ twins share all their genetic sequence but DZ twins share $50 \%$ of genes on average, the higher resemblance between MZ twins (as compared to DZ twins) indicates the heritability of the phenotype. Besides, the twin study also helps decouple environmental influences from common family and social environment (i.e., twin similarity not accounted for by genetic effects) and from personal events (twin differences not due to genetic differences) [8]. In addition, because childhood is a critical period for psychosocial development [9], studies on child twins help depict genetic and environmental influences on the developmental trajectory of conformity.

To examine whether conforming behavior is heritable, previous studies used questionnaires to survey twins' subjective experiences with conformity [10-13]. In this study, we directly measured twins' conforming behavior in a real social situation. Specifically, we created a social episode in which twins were asked to choose a pen from a group of five pens as a gift [5] (Figure 1(a)). Among the five pens, there was one pen with a different color than the rest. And twins had to make a choice between a pen whose color was just like other pens' color (i.e., the majority) and a pen whose color was different from the rest (i.e., the minority). Because conforming behavior is likely induced not only by the pressure from peers but also by the label that objects are associated with (i.e., majority versus minority) even then explicit pressure is absent or normative responses are not immediately obvious [5], the preference for conformity or uniqueness is manifested through the very action of choice of pens. If conformity is heritable, we expect that $\mathrm{MZ}$ twins were more similar in their choice of pens than DZ twins.

(a)

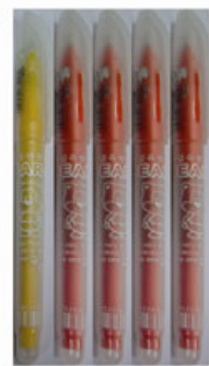

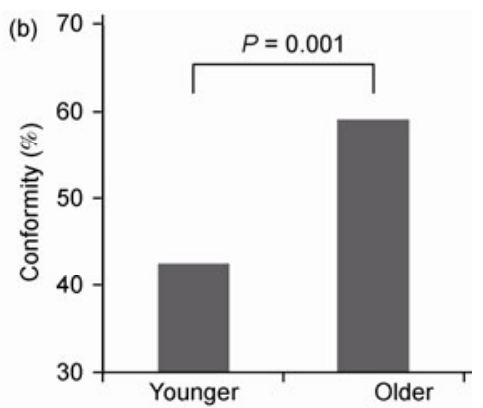

Figure 1 Stimuli and the development of conformity. (a) Pens used in the study. Pens were black-ink ball-point pens with four different barrel colors. Except the barrel color, the pens were essentially the same. Five pens were kept as a group, with one pen in a different color from the rest. The pen with the unique color was labeled as the minority; whereas the rest were labeled as the majority. (b) Conformity increases with age. The older children showed a significant higher preference in choosing pens characterized by the majority color than the younger children. The $y$ axis is the percentage of participants in choosing pens with the majority color.

\section{Methods}

\subsection{Participants}

Three hundred and ninety-nine participants (age 7-19, mean $=12.8, \mathrm{SD}=2.67$ ) were recruited from elementary and middle schools in Beijing, China. They consisted of 107 pairs of MZ twins (age 7-19, mean = 12.9, SD = 2.67), 74 pairs of DZ twins (age 7-18, mean $=12.2, \mathrm{SD}=2.23$ ), and 37 singletons. The zygosity of the twins was determined by a questionnaire on the physical resemblance, which has over 95\% accuracy in predicting blood-typed zygosity of twins [8], and was further validated by the number of placenta where necessary. The singleton participants were not included in genetic analyses. All participants were carefully screened to ensure that they did not have a history of extreme pregnancy/birth, or cognitive/neurological disorders. In addition, 162 college students (age 20-24, mean $=22.3$, $\mathrm{SD}=0.89$ ) participated in an experiment assessing the validity of the implicit measure used in the twin study. The study was approved by the institutional review board of Beijing Normal University. Prior to testing, written informed consent was obtained from the participants and/or from their parents or guardians.

\subsection{Procedure}

The participants were tested individually at their schools by trained experimenters. After a series of computer-based behavior tests, the participants were given a pen as a gift, along with other compensations. The pens were black-ink ball-point pens with four different barrel colors: orange, blue, green, and yellow. Except for the barrel color, the pens were essentially the same (e.g., the same design and the same quality). Before the test, five pens were kept in a box, and among the five, there was only one pen with a different color from the rest. Because in the pilot study we did not find excessive preference to one particular color, the two colors of the pens in a trial were randomly selected from the four colors, with the combinations of the colors being approximately balanced. During the test, the experimenter took all five pens from the box as naturally as possible, and the participant was asked to take one pen as a gift. When the participant made a choice, the experimenter recorded the choice. The change of the choice was not allowed. To avoid social pressure from peers and the possibility that MZ twins may interact in social situations more frequently than DZ twins [14], twins were tested individually in a quiet room where there were only one experimenter and one participant. Therefore, participants were unable to know the other's choice at the time of testing. In addition, different combinations of colors were presented to a twin pair to rule out possible confounding factors such as twins' shared preference on color.

To assess the validity of the implicit measure, we examined whether individuals who chose a pen with the majority 
color were indeed inclined to show conformity in their daily activities. To do this, a new population of singleton college students was tested on the pen-choosing task along with a statement on their daily conforming behavior. The statement, "I don't mind bragging about my talents and accomplishments," was selected from Big Five Personality Inventory (NEO Personality Inventory, Revised) [15]. The participants were asked to report the extent to which that their daily behavior matched the statement in a five-point Likert scale (1: strongly disagree; 5: strongly agree).

\subsection{Data analysis}

If a participant preferred conformity, he or she would choose a pen with the majority color. Thus, the amount of conformity in the population tested was calculated as the percentage of participants who chose the pen with the majority color [5]. In addition, to demonstrate the developmental trajectory of conformity, all child participants (i.e., age between 7 and 19), including the singleton participants, were divided into two age groups: the younger children group (242 participants, age $7-13$, mean $=11.0, \mathrm{SD}=1.46$ ) and the older children group (157 participants, age 14-19, mean $=15.6, \mathrm{SD}=1.53$ ). A stricter test of the relation between conforming behavior and age was performed by a logistic regression analysis with age being a continuous variable.

To investigate possible genetic effects on conformity, the concordance rate (i.e., the resemblance in choosing pens between twins) was calculated as the percentage of twin pairs who made the same choice (i.e., both choosing pens either with the majority color or with the minority color). Similarly, the discordance rate was calculated as the percentage of twin pairs who made different choices. Clearly, the sum of the concordance rate and discordance rate was $100 \%$. Nonparametric Chi-square tests were used to test whether the uniqueness of pens significantly affected the choice between genders, between age groups (i.e., younger versus older children), and between zygosity groups (i.e., MZ versus DZ twins).

To estimate the proportions of variance in conformity accounted for by genetic and environmental influences, liability-threshold model-fitting analyses were performed with OpenMx [16]. In addition, the genetic and environmental components were tested for heterogeneity across age groups of child participants to examine whether their contributions varied during the development. Specifically, the twin data from the two age groups were first simultaneously analyzed in one model with different parameter estimates for the two age groups (i.e., unconstrained). Next, the parameter estimates for the two age groups were treated as being equal (i.e., constrained). By subtracting the negative log likelihood for the constrained model from that for the unconstrained model, we examined quantitative differences in the fit between the two models with means of likelihood-ratio tests.

\section{Results}

\subsection{Conformity increases with age}

First, we used a logistic regression analysis to examine whether conformity changed during the psychosocial development, with age as an independent variable and children' choices of pens as a dependent variable. We found that the conforming behavior was correlated with age, with older children were more likely to choose the pen with the majority color (Wald $\chi^{2}(1)=9.71, P=0.002$ ). To clearly demonstrate the increase of conformity during the development, we split the children into a younger children group (mean age $=11.0$ ) and an older age group (mean age $=15.6$ ) Similarly, a Chi-square test replicated the regression analysis, with the older children (59.2\%) showing a significantly higher preference in choosing the pen with the majority color than the younger children $(42.6 \%)\left(\chi^{2}(1,399)=10.6\right.$, $P=0.001$ ) (Figure 1(b)). The observed increase in conformity was unlikely accounted for by the color of pens, because a two-way interaction of choice (majority versus minority) by color of pens (four different barrel colors) was not significant $\left(\chi^{2}(3,399)=1.14, P=0.77\right)$. Besides, there was no gender effect on the conforming behavior (boys: $46.1 \%$, girls: $\left.53.9 \% ; \chi^{2}(1,399)<1\right)$.

\subsection{The genetic influence on conformity is essentially zero}

To decouple the environmental and genetic influences on conformity, we calculated the concordance rate in $\mathrm{MZ}$ twins and DZ twins respectively. We found that there was no significant difference in the concordance rate between the $\mathrm{MZ}$ twins $(57.0 \%)$ and the DZ twins $(58.1 \%)\left(\chi^{2}(1,181)<1\right)$, suggesting that there is no genetic component in conformity. The lack of genetic influence is unlikely due to the fact that we included different-sex DZ (DSDZ) twins in the analysis. After excluding the DSDZ twins from the analysis, the concordance rate of the same-sex DZ twins (concordance rate $=53.5 \%$ ) was not significantly lower than that of the $\mathrm{MZ}$ twins either $\left(\chi^{2}(1,150)<1\right)$. In addition, the MZ twins did not show more conforming behavior than the DZ twins, as $50.5 \%$ of the MZ twins and $52 \%$ of the DZ twins chose the pen with the majority color $\left(\chi^{2}(1,362)<1\right)$. Therefore, genes apparently play little role in conforming behavior.

\subsection{The increase in conformity with age is driven by the shared component}

To decouple environmental influences into shared and nonshared components, we used a liability-threshold model-fitting analysis (ACE model) on the twin data. We found that the heritability of the conformity was essentially zero, 
consistent with the Chi-square test aforementioned. On the other hand, the shared and nonshared components explained $23.2 \%$ and $76.8 \%$ of the variance of the underlying liability distribution in conformity respectively. In addition, although the full model (i.e., the ACE model) was fitted well (goodness of model-fitting, $\chi^{2}(3)=0.47, P=0.92$; Akaike fit indices or AIC $=-220.4$ ), the best-fitting model was one that consisted of only shared and nonshared components (i.e., the CE model) $\left(\chi^{2}(4)=0.47, P=0.98\right.$; AIC $=-222.36$ ). That is, dropping the additive genetic component of variance (A) from the full model did not significantly worsen the fit of the model (likelihood-ratio test, $\Delta \chi^{2}(1)=0, P=1$ ). However, dropping the shared environment component (C) from the $\mathrm{CE}$ model significantly worsened the fitting (i.e., $\mathrm{E}$ model, $\left.\Delta \chi^{2}(1)=4.02, P=0.04\right)$. Therefore, both shared and nonshared experiences play a sizable role.

To further investigate which environmental component, shared or nonshared, is the primary source of the increase in conformity during the development, we used the univariate heterogeneity twin analysis model to estimate the change of the contribution of shared and nonshared components to conformity during the development. As expected, in both age groups, the estimated heritability of the conformity was zero, suggesting that age did not influence the heritability. By contrast, we found that the variance in the conforming behavior explained by the shared component was significantly larger in the older children group $(26.3 \%)$ than that in the younger children group $(16.0 \%)$, as forcing parameter estimates to be equal for the two age groups significantly worsened the fit of the model $\left(\Delta \chi^{2}(3)=9.03, P=0.03\right)$ (Figure 2). That is, the increase in conformity during the development is likely accounted for by the increased contribution of the shared environmental component.

\subsection{The pen-choosing task is a valid measure of con- formity}

One possible interpretation of the lack of genetic component in conformity is that the implicit measure used in this study did not measure conforming behavior properly. This alter-

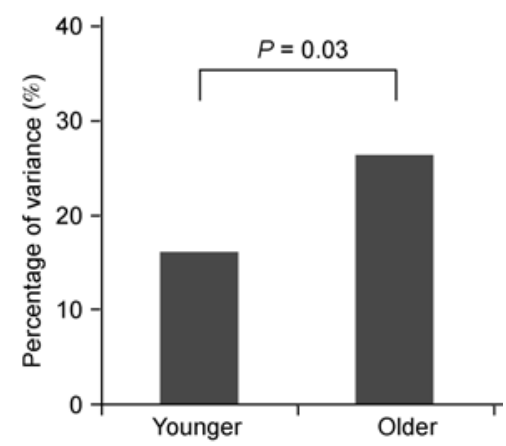

Figure 2 Shared environmental influence on conformity. The contribution of the shared environmental component to conformity increases with age. The $y$ axis shows the percentage of variance in conformity accounted for by the shared component.

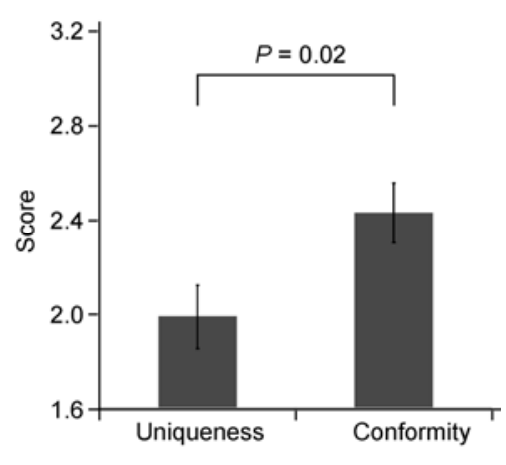

Figure 3 The association between the implicit measure and daily conforming behavior. Individuals who chose a pen with the minority color (i.e., uniqueness) were more likely to report a higher agreement with the statement that "I don't mind bragging about my talents and accomplishments" than those who chose a pen with the majority color (i.e., conformity). The $y$ axis shows the level of agreement on the statement, and the lower the value the higher degree of agreement. The error bar denotes \pm 1 S.E.M. (Standard Error of Mean).

native was rejected in a test on a new population of singleton participants, who were asked to explicitly report their daily activities on conformity. Indeed, individuals who chose a pen with the minority color (i.e., uniqueness) reported that they were likely to stand out in the public than those who chose a pen with the majority color (i.e., conformity) $(t(160)=-2.41, P=0.02)$ (Figure 3$)$. That is, the way of choosing pens in the implicit test predicted explicit conforming behavior in real social situations.

\section{Discussion}

In a social situation, individuals often follow others' action to achieve an accurate interpretation of their circumstances, to obtain approval from others and to maintain a favorable self-esteem. Here we probed the possible genetic origin with the behavioral genetics approach. Two new findings have been observed. First, there was no sign of the heritability of conformity. Second, both shared and nonshared environmental components played a significant role in shaping individuals' conforming behavior. More importantly, the portion of shared component in conformity increased with age, which may account for the increase of conformity during the psychosocial development observed in the participants. Therefore, we are not born but raised to follow the crowd.

The child participants tested in this study aged from 7 to 19, which is a critical period for the psychosocial development. During this period, children start to manifest autonomy-related behaviors to develop personal identity [9], and either conforming or deviating from others' actions helps provide individuals with a sense of belonging or uniqueness, respectively $[5,17,18]$. Specifically, to maintain positive self-assessments on their personal identity, children usually conform to valued groups during the psychosocial 
development $[19,20]$. This idea is supported by our observation that the conformity increased with age, with older children showed a higher preference in selecting pens with the majority color than younger ones.

The ACE model further showed that both shared and nonshared environmental components contribute to the individual differences in conformity. Importantly, the shared component is likely the primary source in shaping conformity during the psychosocial development [11], because the increase in conformity during the development is accompanied by the increased proportion of the shared component in conformity. In China, where our study was conducted, conformity is prized as harmony and connections among people, and thus defines the concept of self [5,21,22]. Indeed, parents in China prefer to adopt an authoritarian type of parenting practice: a low level of warmth, a low level of autonomy granting and a high level of behavioral control [23], as compared with parents in individualistic countries [24-27]. As a result, adults who have authoritarian parents are more likely to emphasize conformity values [4]. Therefore, the common family and culture environment has important ramifications for the development of conformity.

On the other hand, it is not surprising that conformity is not heritable. Other social behaviors emphasizing on interpersonal interactions [28], such as romantic love [29] and physical attractiveness [30], are not heritable either. Similarly, conforming behavior, which solicits others' approvals, requires intensive interpersonal interactions. However, the type of the approval differs across social situations (e.g., with parents versus with peers) [4,31] and across cultures (e.g., collectivistic versus individualistic countries) [5,21]; therefore, the concept of conformity is assigned with very different meanings. For example, the same behavior may represent conformity in Western culture but harmony in East Asian culture [5]. Because of its complexity, the predisposition of being conformists or nonconformists is unlikely encoded in genes.

However, studies based on Eysenck Personality Questionnaire [11], Drinking Motives Questionnaire [10] and Rokeach Value Survey [12] show that conformity is instead heritable. We argue that such inconsistence may reflect the difference between the self-reports used in their studies and the implicit measure used in this study. It has been suggested that implicit measures predict behaviors better than self-reports from questionnaires [32], especially on sensitive issues related to social norms such as conformity and compliance [33,34]. In addition, the heritability of conformity identified with the self-reports was relatively small (e.g., 6\%) in some population tested [11], and a recent twin study based on Drinking Motives Questionnaire even failed to identify the heritibity of conformity [13], consistent with our finding. Future studies with other types of situational experiments (e.g., Asch conformity task) on a larger sample of twins may help further examine the heritability of conformity.

In sum, our study demonstrates a strong role of nurture in shaping our ability in balancing between our internal judgment and external pressures to do otherwise. Such complexity present in the conformity and other social activities has been proposed to be the primary drive for the evolution of the human brain [35]. Therefore, further studies with functional magnetic resonance imaging techniques may help reveal how the social influences from the common family and culture environment shape the brain and thus social behaviors during the psychosocial development.

We thank XU Rui, LUO Yu, TONG Yi for data collection; and QIAO Shuping, LIANG Wei and LI Tian for help with registering twins. This work was supported by the National Natural Science Foundation of China (31230031, 91132703) and the National Basic Research Program of China (2010CB833903).

1 Burke C J, Tobler P N, Schultz W, et al. Striatal bold response reflects the impact of herd information on financial decisions. Front Hum Neurosci, 2010, 4: 48

2 Jolles J W, de Visser L, van den Bos R. Male wistar rats show individual differences in an animal model of conformity. Anim Cogn, 2011, 14: 769-773

3 Klucharev V, Hytonen K, Rijpkema M, et al. Reinforcement learning signal predicts social conformity. Neuron, 2009, 61: 140-151

4 Kasser T, Koestner R, Lekes N. Early family experiences and adult values: A 26-year, prospective longitudinal study. Pers Soc Psychol Bull, 2002, 28: 826-835

5 Kim H, Markus H R. Deviance or uniqueness, harmony or conformity? A cultural analysis. J Pers Soc Psychol, 1999, 77: 785-800

6 Cialdini R B, Goldstein N J. Social influence: Compliance and conformity. Annu Rev Psychol, 2004, 55: 591-621

7 Fehr E, Fischbacher U. Social norms and human cooperation. Trends Cogn Sci, 2004, 8: 185-190

8 Plomin R, DeFries J C, McClearn G E, et al. Behavioral Genetics. New York: Worth, 2008

9 Steinberg L, Silverberg S B. The vicissitudes of autonomy in early adolescence. Child Dev, 1986, 57: 841-851

10 Agrawal A, Dick D M, Bucholz K K, et al. Drinking expectancies and motives: A genetic study of young adult women. Addiction, 2008, 103: 194-204

11 Pergadia M L, Madden P A, Lessov C N, et al. Genetic and environmental influences on extreme personality dispositions in adolescent female twins. J Child Psychol Psyc, 2006, 47: 902-909

12 Schermer J A, Vernon P A, Maio G R, et al. A behavior genetic study of the connection between social values and personality. Twin Res Hum Genet, 2011, 14: 233-239

13 Kristjansson S D, Agrawal A, Littlefield A K, et al. Drinking motives in female smokers: Factor structure, alcohol dependence, and genetic influences. Alcoho Clin Exp Res, 2011, 35: 345-354

14 Constantino J N, Todd R D. Genetic structure of reciprocal social behavior. Am J Psychiat, 2000, 157: 2043-2045

15 White J K, Hendrick S S, Hendrick C. Big five personality variables and relationship constructs. Pers Indiv Differ, 2004, 37: 15191530

16 Boker S, Neale M, Maes H, et al. Openmx: An open source extended structural equation modeling framework. Psychometrika, 2011, 76: 306-317

17 Blanton H, Christie C. Deviance regulation: A theory of action and identity. Rev of Gen Psychol, 2003, 7: 115-149

18 Nail P R, MacDonald G, Levy D A. Proposal of a four-dimensional model of social response. Psychol Bull, 2000, 126: 454-470

19 Brewer M B, Roccas S. Individual Values, Social Identity, and Opti- 
mal Distinctiveness. In: Individual Self, Relational Self, Collective Self. Philadelphia: Psychology Press, 2001. 219-237

20 Pool G J, Wood W, Leck K. The self-esteem motive in social influence: Agreement with valued majorities and disagreement with derogated minorities. J Pers Soc Psychol, 1998, 75: 967-975

21 Bond R, Smith P B. Culture and conformity: A meta-analysis of studies using asch's $(1952 b, 1956)$ line judgment task. Psychol Bull, 1996, 119: 111-137

22 Zhang L, Zhu H, Xu M, et al. Selective impairment in recognizing the familiarity of self faces in schizophrenia. Chin Sci Bull, 2012, 57: $1818-1823$

23 Steinberg L, Elmen J D, Mounts N S. Authoritative parenting, psychosocial maturity, and academic-success among adolescents. Child Dev, 1989, 60: 1424-1436

24 Chiu L H. Child-rearing attitudes of Chinese, Chinese-American, and Anglo-American mothers. Int J Psychol, 1987, 22: 409-419

25 Dornbusch S M, Ritter P L, Leiderman P H, et al. The relation of parenting style to adolescent school performance. Child Dev, 1987, 58: $1244-1257$

26 Liu M, Guo F. Parenting practices and their relevance to child behaviors in Canada and China. Scand J Psychol, 2010, 51: 109-114

27 Liu M M, Chen X Y, Rubin K H, et al. Autonomy- vs. Connectedness-oriented parenting behaviours in Chinese and Canadian mothers.
Inter J Behav Dev, 2005, 29: 489-495

28 Kim C K, Lee K, Chung M. A renormalization group approach to crowd psychology and inter-group coupling in social many-body systems. Chin Sci Bull, 2012, 57: 247-252

29 Waller N G, Shaver P R. The importance of nongenetic influences on romantic love styles-A twin-family study. Psychol Sci, 1994, 5: 268-274

30 Lykken D T, Tellegen A. Is human mating adventitious or the result of lawful choice-A twin study of mate selection. J Pers Soc Psychol, 1993, 65: 56-68

31 Fuligni A J, Eccles J S. Perceived parent-child relationships and early adolescents orientation toward peers. Dev Psychol, 1993, 29: 622632

32 Greenwald A G, Poehlman T A, Uhlmann E L, et al. Understanding and using the implicit association test: Iii. Meta-analysis of predictive validity. J Pers Soc Psychol, 2009, 97: 17-41

33 Kraus S J. Attitudes and the prediction of behavior-A metaanalysis of the empirical literature. Pers Soc Psychol Bull, 1995, 21: 58-75

34 Towles-Schwen T, Fazio R H. Automatically activated racial attitudes as predictors of the success of interracial roommate relationships. J Exp Soc Psychol, 2006, 42: 698-705

35 Byrne R W, Bates L A. Sociality, evolution and cognition. Curr Biol, 2007, 17: R714-723

Open Access This article is distributed under the terms of the Creative Commons Attribution License which permits any use, distribution, and reproduction in any medium, provided the original author(s) and source are credited. 